\title{
Micro-scale thermal analysis of organic and polymeric materials using cooled and un-cooled infrared cameras
}

\author{
by J. Morikawa*, E. Hayakawa* and T. Hashimoto**
}

*Dept. of Organic \& Polymeric Materials, Tokyo Institute of Technology, Ookayama 2-12-1, S8-29, Meguro-ku, Tokyo 152-8550, Japan, morikawa.j.aa@m.titech.ac.jp, hashimoto.t.ae@m.titech.ac.jp

**ai-Phase, Co., Ltd., Kami-osaki, 2-15-19, Shinagawa-ku, Tokyo 141-0021, Japan

\begin{abstract}
Thermal imaging with a spatial resolution of micro-scale $(\sim 10 \mu \mathrm{m})$ is required in increasing numbers of applications of thermal functioning materials, monitoring of small temperature changes and its gradients in localized micro-scale area. The emissivity corrected thermal imaging using a real time direct impose-signal system and the method to obtain the afterimage of thermal wave with a newly equipped instrument are presented. The applicability of uncooled micro-bolometer (thermal detector) to the micro-scale thermal analysis on phase transitions is either discussed in comparison with the photon detector.
\end{abstract}

\section{Introduction}

In order to visualize the material's thermal phenomena with a spatial resolution $\sim 10 \mu \mathrm{m}$ and in a time scale $10 \mathrm{~ms}$, optics, precise temperature control systems and some mathematical algorithms have been developed. The measurement is done preferably under the dynamic temperature field such as a constant rate heating / cooling and a temperature modulation. The latent heat generation and dissipation at the minus temperatures during freezing of biological plant and animal cells are visualized that clarifies the thermal diffusion effect on crystallization and the vitrification [1] - [5]. On-lamellae thermal analysis of $n$-alkane visualizes the early stage of anisotropic lamella formation and the difference of thermal propagation in crystallizations and the rotator phase transitions [6]. The crystallization front of polymeric spherulite of poly(ethylene oxide) visualizes the temperature rise of $\sim 100 \mathrm{mK}$ [7]. The basic results of lock-in thermography visualizing in-plane thermal diffusivity and the thermal interface are presented with a modulated spot heating method using a diode laser that is used to generate a thermal wave inside the specimen [8] .

In spite of the improvement of the resolution of time and space with the techniques developed as above, the procedure for emissivity correction is still important, especially for the thermal phenomena including phase transitions. The emissivity change during phase transitions is not a simple function of temperature.

In order to calibrate the intensity of all pixels in FPA into temperature, the measured temperature data is directly imposed to the intensity signal. This approach to calibrate the emissivity is one of the purposes of this paper.

\section{Experimental}

\subsection{Measuring setup: General}

The measuring setup is composed of a direct signal imposing system and a laser drive control based on the precise and dynamic temperature control. The chamber for sample-holder is replaced according to the required measuring conditions of temperature and atmospheric pressure.

\subsubsection{Optics}

The microscopic lens is originally developed considering the spectral band wavelength and the angle of the view of the sensor. The value of modulation transfer function (MTF: the modulus of the OTF) is calculated as a function of the spatial frequency in cycles per $\mathrm{mm}$ and the image height. The achromatic lens system must be designed for LWIR FPA. The diffraction limit of the lens for the object side is listed in Table I, $11 \mu \mathrm{m}$ for LWIR thermal FPA, and $4.3 \mu \mathrm{m}$ for MWIR photon FPA.

The total length-size of the IR-FPA + optics using uncooled thermal FPA is less than $10 \mathrm{~cm}$. The anti reflection coating on the achromatic lens groups, which is composed of different materials suited to spectral band of LWIR, are chosen for the optimum MTF and the minimum distortion. Spatial resolution was examined with a standard USAF 1951 test pattern. The half pitch size of the frequency corresponding to $10-14 \mu \mathrm{m}$ becomes distinguishable using VOx FPA. 
Table I

\begin{tabular}{|c|c|c|c|c|c|}
\hline & & VOx micro-bolometer & VOx & $\mathrm{InSb}$ & InSb \\
\hline \multirow[t]{7}{*}{ FPA } & spectral response & $7.5-13.5 \mu \mathrm{m}$ & $7.5-13.5 \mu \mathrm{m}$ & $3-5 \mu \mathrm{m}$ & $3-5 \mu \mathrm{m}$ \\
\hline & pixel pitch & $25 \mu \mathrm{m}$ & $17 \mu \mathrm{m}$ & $30 \mu \mathrm{m}$ & $25 \mu \mathrm{m}$ \\
\hline & pixel number & $240 \times 320$ & $480 \times 640$ & $320 \times 256$ & $640 \times 512$ \\
\hline & area size & $6 \times 8$ & $8.16 \times 10.88$ & $9.6 \times 7.68$ & $16 \times 12.7$ \\
\hline & opposite angle & 10 & 13.6 & 13.65 & 20.43 \\
\hline & MTF & $1 / 0.025 / 2=20 \mathrm{lp} / \mathrm{mm}$ & $1 / 0.017 / 2=30 \mathrm{lp} / \mathrm{mm}$ & $1 / 0.03 / 2=17 \mathrm{lp} / \mathrm{mm}$ & $1 / 0.025 / 2=20 \mathrm{lp} / \mathrm{mm}$ \\
\hline & sensitivity & $50 \mathrm{mK} @ \mathrm{f} / 1$ & $50 \mathrm{mK}$ & $25 \mathrm{mK}$ & $20 \mathrm{mK}$ \\
\hline \multirow[t]{6}{*}{ OPTICS } & resolution (object side) & $14 \mu \mathrm{m}($ at $\lambda=10 \mu \mathrm{m})$ & $11.1 \mu \mathrm{m}($ at $\lambda=10 \mu \mathrm{m})$ & $4.29 \mu \mathrm{m}($ at $\lambda=5 \mu \mathrm{m})$ & $4.29 \mu \mathrm{m} \quad($ at $\lambda=5 \mu \mathrm{m})$ \\
\hline & magnification & 2.5 & 2.472 & 10 & 8.33 \\
\hline & object size & $2.40 \times 3.20$ & $3.264 \times 4.352$ & $0.96 \times 0.768$ & $1.92 \times 1.524$ \\
\hline & opposite angle & 4 & 5.44 & 1.229 & 2.451 \\
\hline & NA object side & 0.435 & 0.555 & 0.706 & 0.706 \\
\hline & WD & $22 \mathrm{~mm}$ & $15 \mathrm{~mm}$ & $14.38 \mathrm{~mm}$ & $15.4 \mathrm{~mm}$ \\
\hline
\end{tabular}

\subsubsection{Measuring system}

The measuring instrument, including the signal imposing system is designed with the components as follows; A: Signal (NTSC, LVDS) capturing part, B: Superimposer of video signal, C: Timing trigger generator, D: Synchronous IR camera with InSb and VOx FPA sensors, E: High-precision / high-speed temperature controller, F: Acutuator and laser drive, G: Stage scanners. A handy size design is realized when VOx FPA is applied (Fig. 1).

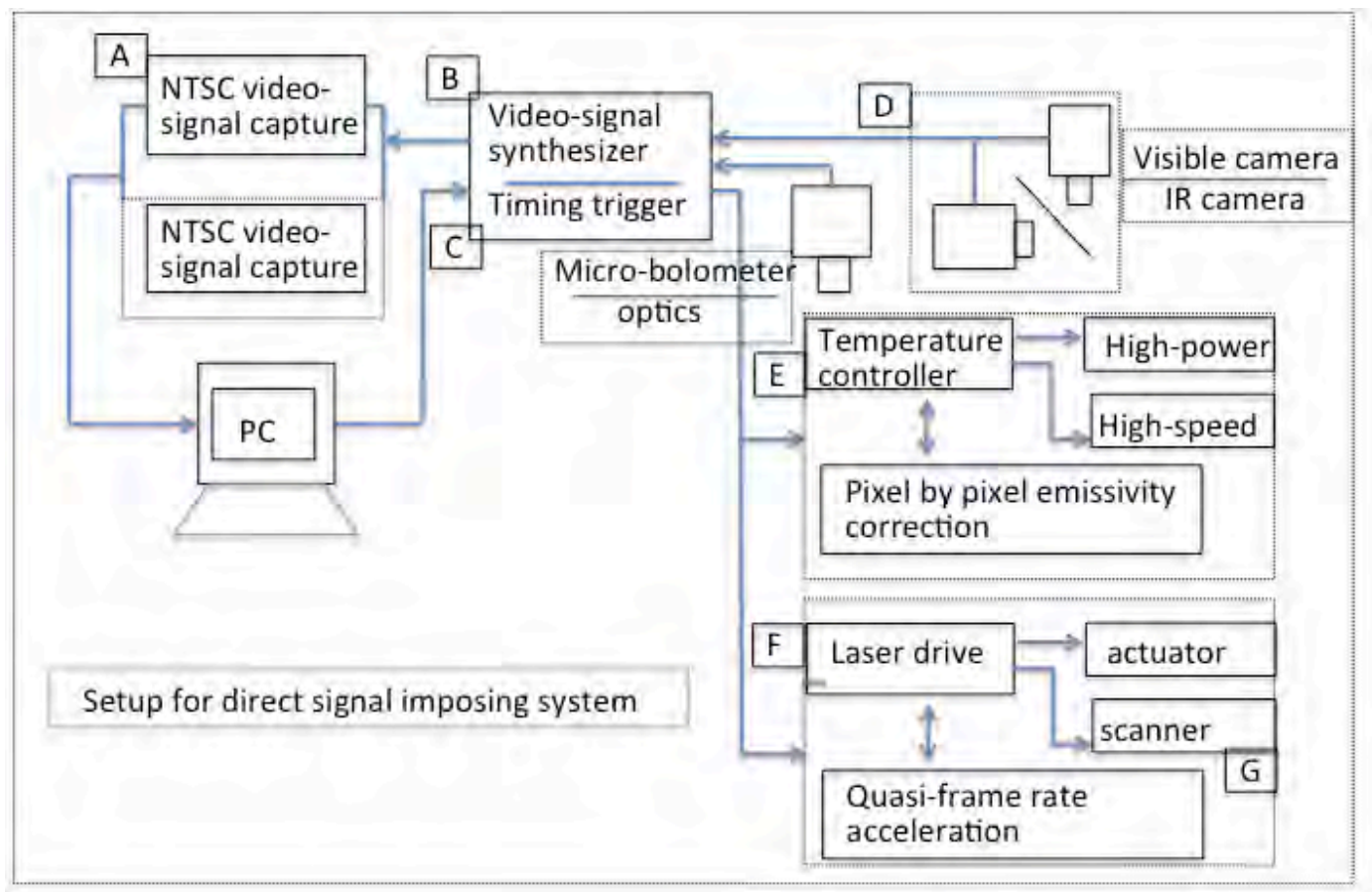

Fig. 1 Measuring setup.

\subsubsection{Video-signal Superimpose}

The brightness data is serial, synchronized to the data clock, with16 bit per1 pixel. The frequency of data clock is $70 \mathrm{MHz}$ or $200 \mathrm{MHz}$. The synchronized signal of VSYNC, and HSYNC of NTSC signal are included in Data sync signal. 
The signal synthesizing function replaces the data in the imposed area from the camera input (data clock, data out, and data sync), into the imposed pixel data as a data output. The signal synthesizing process is schematically shown in Fig. 2.

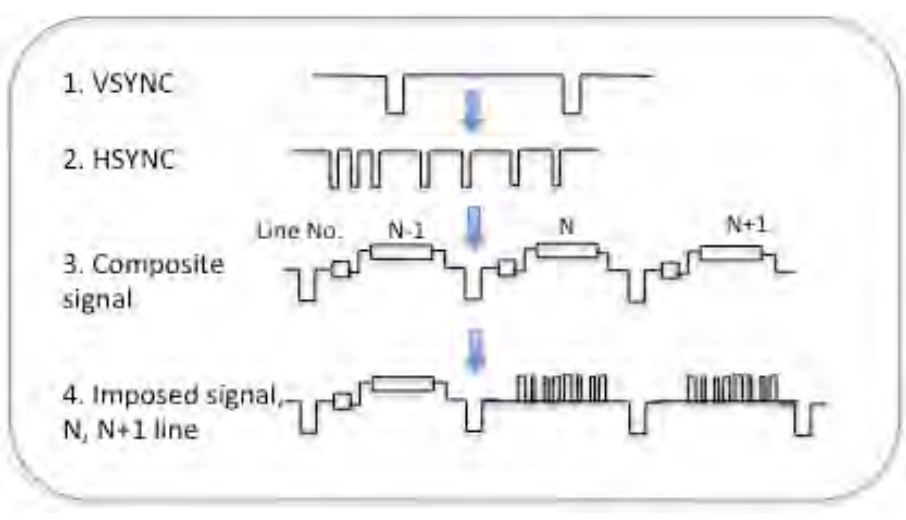

Fig. 2 Scheme of the video-signal synthesizer

The direct signal imposed system is composed of the video-signal synthesizer, the timing trigger, and NTSC video-capturing hardware connected to IR camera data processing system. The video signal synthesizer enables to record a direct digital signal monitoring of temperature or positioning data, simultaneously, that is measured with different sensor systems. The encoded digital signal data embedded in each image is decoded to read out the data. The protocol to encode/decode the measured data is originally defined. In order to observe a heat flow under a temperature scan, a temperature controller is settled and operated with a temperature precision $0.1^{\circ} \mathrm{C}$. The mixed signals of IR camera and the imposed data are applied to the all pixel emissivity corrections and the pseudo-acceleration of the periodical thermal phenomena.

\subsubsection{Laser drive control and scanning micro-stage}

The laser drive for generating a modulated spot heating with $630 \mathrm{~nm}$ diode laser and the xy positioning actuator are triggered with a timing signal synchronous to the NTSC image capturing (Fig. 3). The video signal synthesizer enables to record a direct signal monitoring of temperature or positioning data, simultaneously, that is measured with different sensor systems. The imposed data are applied to the pixel emissivity corrections at one-time under heating/cooling temperature scan. The Peltier-type dual heater is set to operate a heating and cooling scan of the specimens.

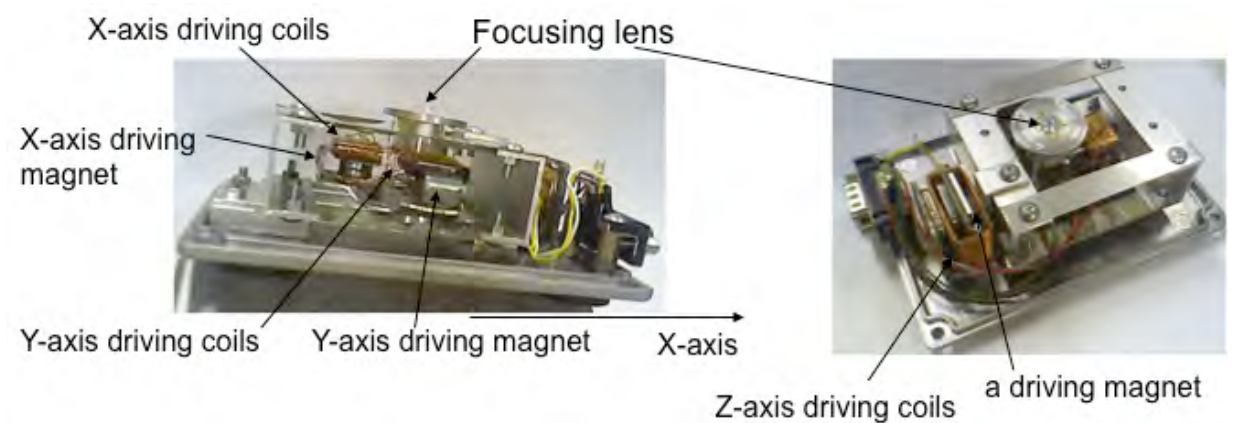

Fig. 3 XYZ-micro-scan stage and the laser drive.

\subsubsection{Pseudo-acceleration}

In order to improve the time-scale resolutions for the periodic thermal event, such as a modulated spot heating, the algorithm for "pseudo-acceleration" is developed. The scheme of the principle is described in Fig. 2.

Fig. 2 shows that the capturing timing of camera and the periodic heat source driving are not synchronized. There exists a variable time delay. However, if the image captured in one cycle is re-ordered over some cycles, the time resolution is apparently improved. It contributes to reduce the noise by integrating the multiple image data, keeping a time resolution. (Usually, the exposure time is increased in order to reduce the noise, which damages the time resolution.) 
This method is effective especially to a limited frame rate for capturing the images caused by a thermal response time $\tau_{T}$ of the thermal detector remains, (defined as $\tau=C$ / $G$ where $C$ is the heat capacity of the pixel and $G$ is the sum of the thermal conductances of all the heat loss mechanisms that remove heat from the pixel).

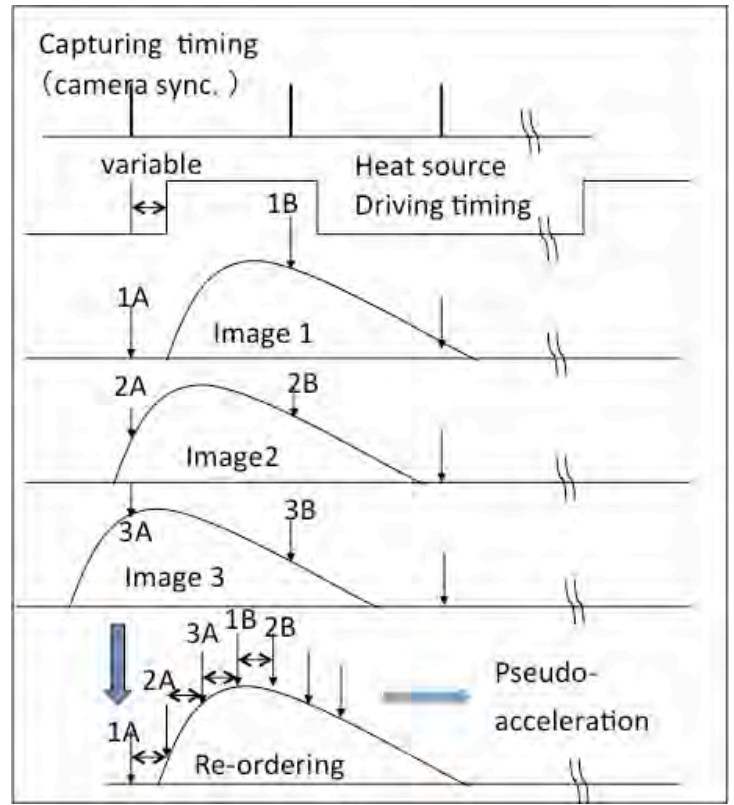

Fig. 3 Scheme of the principle of "pseudo-acceleration) of periodic thermal events.

\subsubsection{Pixel by pixel temperature - emissivity conversion}

Pixel by pixel temperature-emissivity conversion is carried out using the video-signal superimpose technique (2.1.3). The emissivity of industrial materials and biological tissues are usually un-homogeneous, which have the different temperature dependences depending on each pixel. By such reasons, it is difficult to convert the emissivity into absolute temperature operated in the non-steady state temperature field, such as under a temperature scan for observing the phase transitions or an input of modulated heat source to observe the thermal diffusion. The precise conversion is realized using the superimposed signal, because of the high-resolution of the spatial and time directions.

The system visualizes the thermal phenomena under a temperature scan and a modulated heat input. The temperature-scan drive, and the laser scan drive are linked to the camera video capturing system separately. For thermal analysis, the relationship of the brightness and imposed temperature data is obtained during the measurement itself. For the conversion of brightness data into temperature, Stephan-Boltzmann law is used after subtracting the dark current noise.

\section{Results}

\subsection{Pixel by pixel temperature calibration using a direct imposed digital temperature signal}

\subsubsection{InSb FPA}

Figure $4 \mathrm{~A}-\mathrm{C}$ show the thermal imaging of biological tissues of onion-skin cells captured by InSb FPA in a cooling scan with the method of pixel by pixel temperature calibration with the imposed temperature signal. The emissivity is corrected with the imposed signal (Figure 1a shows the uniformity in temperature after the correction) and the calibrated temperature is shown as a contrast of light and shade. In Fig. 4 shortly after the surface water becomes frozen, the freezing of cell starts in one cell by one cell, and because of the localized temperature rise caused by thermal diffusion from the freezing cell next to another one, the freezing proceeds in a random way. 


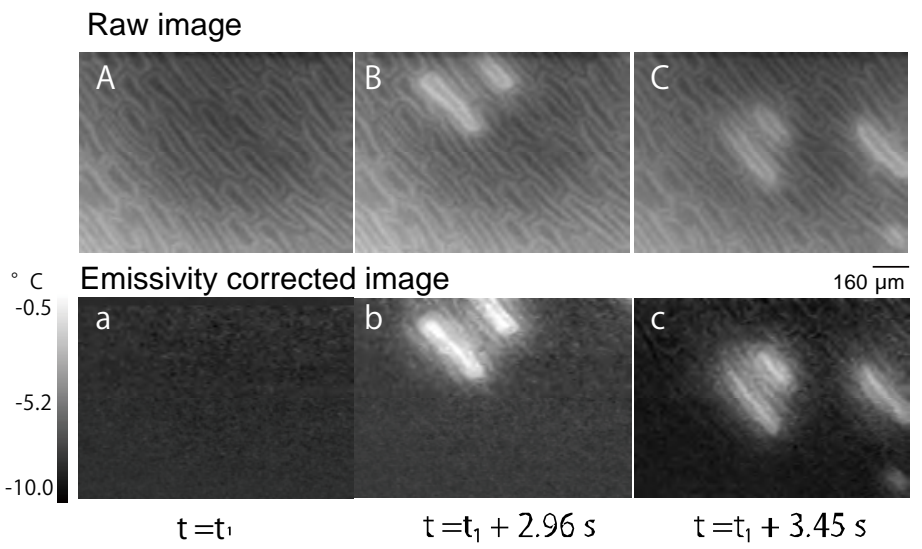

Fig. 4 Sequence of the original thermal image $(A, B, C)$ and the emissivity corrected image $(a, b, c)$ of freezing biological cells at minus temperatures under a cooling scan observed with InSb IR FPA (25 $\mu$ m detector pitch) equipped with a $\times 10$ magnification micro-lens. After the emissivity correction, temperature of each pixel is calibrated. The temperature is shown in the concentration bar in the left. The signal is captured via NTSC in the time interval as follows; $\mathrm{t}=\mathrm{t}_{1}, \mathrm{t}_{1}+2.96 \mathrm{~s}, \mathrm{t}=\mathrm{t}_{1}+3.45 \mathrm{~s}$.

\subsubsection{VOx FPA}

The emissivity corrected thermal image of freezing onion-skin cells captured by a micro-bolometer VOx FPA is shown in Fig. $5 \mathrm{~A}-\mathrm{C}$, together with the temperature profile at a pixel of the intersection in Fig. 5C is shown in Fig. 5D. The cooling rate is $0.15 \mathrm{~K} / \mathrm{s}(9 \mathrm{~K} / \mathrm{min})$. The temperature increase of the main peak is $6.6^{\circ} \mathrm{C}$ in Fig. 6D. The characteristic freezing phenomena of biological plant tissues were reported using the InSb FPA [1,2]. The results in Fig. 5 show that the micro-bolometer is quantitatively applicable to the micro-scale thermal analysis of freezing tissues at minus temperatures. The thermal diffusion over the tissues and the temperature increase caused by the thermal diffusion from the outside is observed in Fig. 5.

\section{Emissivity corrected image}

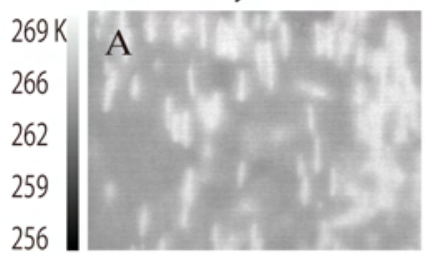

$\mathrm{t}=27.18 \mathrm{~s}$

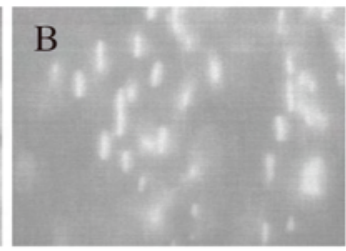

$\mathrm{t}=27.35 \mathrm{~s}$

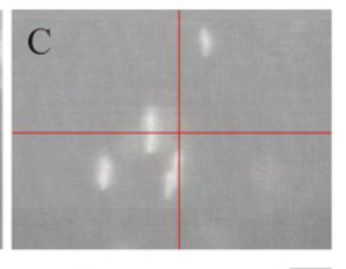

$\mathrm{t}=27.68 \mathrm{~s}$

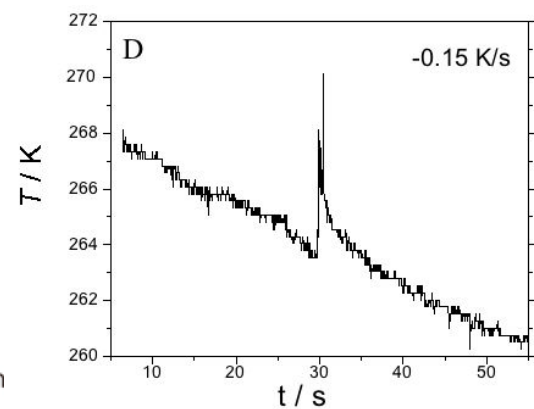

Fig. 5 Sequence of the emissivity corrected thermal image (A, B, C) of freezing onion-skinl cells at minus temperatures under a cooling scan observed with VOx micro-bolometer FPA (17 $\mu \mathrm{m}$ detector pitch) equipped with a $\mathrm{x}$ 2.47 magnification micro-lens. After the emissivity correction, the brightness data of each pixel is converted to the temperature data. The temperature is shown in the concentration bar in the left. The signal is captured via NTSC in the time interval as follows; $t=27.18 \mathrm{~s}, 27.35 \mathrm{~s}, 27.68 \mathrm{~s}$. The temperature profile at a pixel of intersection in Fig. $5 \mathrm{C}$ is shown in Fig. 5D.

\subsection{Pseudo-acceleration of the periodical thermal events}

The testing measurement of the pseudo acceleration of the frame rate during a modulated micro-scale spot heating is examined using the VOx FPA equipped with a micro lens system. Fig. 6 shows the phase shift data measured in the polyimide composite film (uni-axially oriented) at a frequency of $0.97 \mathrm{~Hz}$. The raw data is captured by a rate of 33 $\mathrm{ms} /$ frame, whereas after pseudo acceleration procedure the frame rate is improved as $1.45 \mathrm{~ms} / \mathrm{frame}$. The resolutions of phase and amplitude image (depicted in a contour line in Fig. 6 A-D) are improved with an pseudo-acceleration using a superimposed data. The comparison of phase profile over $\sim 300 \mu \mathrm{m}$ is plotted in Fig. $6 \mathrm{E}$. 


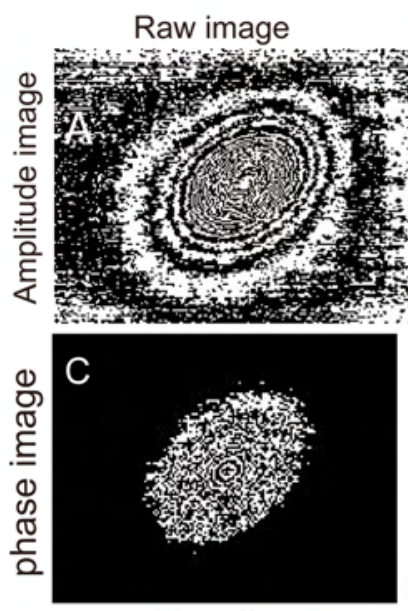

$33 \mathrm{~ms} /$ frame
Pseudo accelerated jmage.

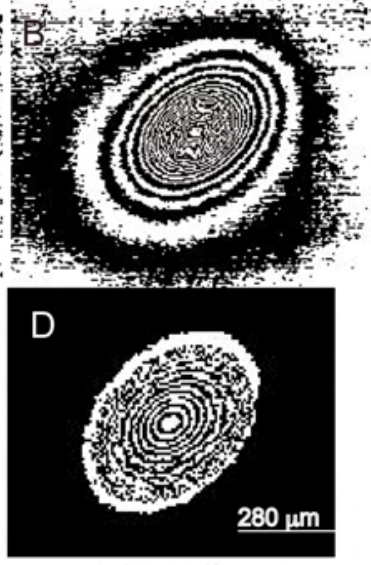

$1.45 \mathrm{~ms} /$ frame

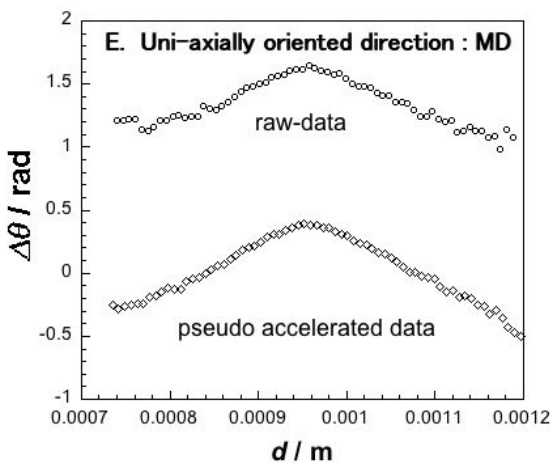

Fig. 6 Comparison of the raw (33 ms/frame) and pseudo-accelerated (1.45 ms/frame) data of a modulated spot heating on uni-axially oriented polyimide film. A, B: Amplitude image, C, D: Phase image, depicted with a contour line, E: Comparison of phase shift of raw and pseudo-accelerated data in the MD direction of the film. The modulated frequency is $0.97 \mathrm{~Hz}$.

\section{Conclusion}

The new techniques originally developed, targeting for the quantitative micro-scale thermal imaging is reviewed. 1. Optics, 2. Superimpose system, and 3. Laser scan drive, are applied to the emissivity-temperature pixel by pixel digital conversion, and the pseudo acceleration of the frame rate. It enhances the spatial and time resolution of InSb and VOx FPA systems, respectively. Combining these techniques are to be applied to the precise and quantitative thermal imaging using cooled and uncooled IR FPA systems.

\section{Acknowledgement}

Support via a grant from Japan Science and Technology Agency (Development of System and Technology for Advanced Measurement and Analysis) is gratefully acknowledged.

\section{REFERENCES}

[1] T. Hashimoto, J. Morikawa, " Two-dimensional thermal analysis on freezing of onion epidermal cell by highspeed infrared microscopic camera," Jpn. J. Appl. Phys. 42, L706-L708 (2003).

[2] J. Morikawa, T. Hashimoto, E. Hayakawa, H. Uemura, "Two-dimensional thermal analysis for freezing of plant and animal cells by high-speed microscopic IR camera," SPIE 5073, 148-153 (2003).

[3] J. Morikawa, T. Hashimoto, K. Yamamoto, J. Ando, "Tow-dimensional thermal analysis for freezing of endothelial cells by high-speed microscopic IR focal plane arrays," SPIE 5697, 282-290 (2005).

[4] J. Morikawa, T. Hashimoto, T. Eto, "Two-dimensional thermal analysis of organic materials by IR thermography" QIRT 2006 Conference,Padva, Italy, 28-30 June, 2006, paper QIRT 2006-036.

[5] C. Pradere, J. Morikawa, J. C. Batsale, T. Hashimoto, "Microscale thermography of freezing biological cells in view of cryopreservation, "Quantitative Infra Red Thermography Journal 6, 37 (2009).

[6] Morikawa, T. Hshimoto, E. Hayakawa, T. Eto, " Two-dimensional thermal analysis of organic materials by microscale thermography" QIRT 2008 Conference, Krakov, Poland, 2-5 July, 2008, paper pp. 397 - 401.

[7] J. Morikawa, E. Hayakawa, K. Ikuo, T. Hashimoto, " Two-dimensional thermal analysis of organic molecular crystals and polymeric spherulites by microscale thermography " SPIE 7661, 76610S-1-9 (2010).

[8] J. Morikawa, T. Hshimoto, E. Hayakawa, T. Eto, R. Li Voti, "Thermal characterization of multi-layer polymer film by IR thermography" QIRT 2006 Conference, Padva, Italy, 28-30 June, 2006, paper QIRT 2006-020. 